\title{
Introduction of a Dedicated Emergency Department MR Imaging Scanner at the Barrow Neurological Institute
}

\author{
(D) M. Buller and (D).P. Karis
}

\begin{abstract}
SUMMARY: Use of advanced imaging in the emergency department has been increasing in the United States during the past 2 decades. This trend has been most notable in CT, which has increased concern over the effects of increasing levels of medical ionizing radiation. MR imaging offers a safe, nonionizing alternative to $C T$ and is diagnostically superior in many neurologic conditions encountered in the emergency department. Herein, we describe the process of developing and installing a dedicated MR imaging scanner in the Neuroscience Emergency Department at the Barrow Neurological Institute and its effects on neuroradiology and the emergency department in general.
\end{abstract}

ABBREVIATION: ED = emergency department

l: the United States, use of advanced imaging in the emergency department (ED) has been steadily increasing during the past 2 decades. ${ }^{1,2}$ This trend has outpaced the increasing number of ED visits, indicating that at least part of this rise is due to increasing demand for these imaging tests. ${ }^{3}$ While this increasing use has been successfully reversed in some centers with education programs, clinical decision support systems, pay-for-performance incentive systems, and other methods, ${ }^{3,4}$ national data continue to demonstrate growth, most notably in CT. ${ }^{1}$ Neuroimaging makes up a substantial portion of this use, constituting a large proportion of total CT imaging ${ }^{3,5}$ and most MR imaging ordered in the ED. ${ }^{2,5}$ These trends have led to increasing concern regarding the rising health care costs and the radiation dose attributable to increasing CT use. MR imaging provides a nonionizing alternative to CT for many neuroimaging indications; however, factors such as availability, scan length, and cost can limit its utility as a practical substitute for CT.

The dedicated Neuroscience Emergency Department at the Barrow Neurological Institute sees $>18,500$ patients per year, with $>2500$ hospital admissions per year for neurologic concerns. This number of patient encounters generates a high volume of

Received January 24, 2017; accepted after revision February 23.

From the Neuroradiology Department, Barrow Neurological Institute, Phoenix, Arizona.

Paper previously presented in part at: Annual Meeting of the Western Neuroradiological Society, October 14-16, 2016; Austin Texas.

Please address correspondence to John P. Karis, MD, Neuroradiology Department, Barrow Neurological Institute, 350 W Thomas Rd, Phoenix, AZ 85013; e-mail: jkaris@sniweb.net

-- Indicates open access to non-subscribers at www.ajnr.org

http://dx.doi.org/10.3174/ajnr.A5210 neuroimaging. In an effort to increase access to MR imaging and create a viable alternative to $\mathrm{CT}$ in this patient population, the Barrow Neurological Institute installed a dedicated ED MR imaging scanner in 2015. Herein, we describe our experience with this process and the effects of a dedicated ED MR imaging scanner on neuroradiology and the emergency department.

\section{Impetus for Change}

On the basis of CT usage data from the early 1990s and extrapolation to 2007 , Brenner and $\mathrm{Hall}^{6}$ estimated that $1.5 \%-2 \%$ of all cancers in the United States could be attributed to radiation from CT studies. Using BEIR VII data and CT head usage data from 2008, Smith-Bindman et $\mathrm{al}^{7}$ estimated that the risk of developing cancer from a CT of the head at age 40 is 1 in 8100 for women and 1 in 11,080 for men. This risk was estimated to double if the age at scanning was halved. Berrington de González et al ${ }^{8}$ suggested that CT head scans performed in the United States in 2007 caused approximately 4000 new cancers. These risks were qualified somewhat in a retrospective review by Sodickson et $\mathrm{al}^{9}$ in 2009, which suggested that while there was only an incrementally increased risk of CT-induced cancer for patients undergoing a limited number of scans, there was significantly increased risk to those undergoing recurrent scanning. Their data demonstrated that $33 \%$ of their cohort of 31,462 patients had undergone $\geq 5$ CT scans during the 22 -year study period and that $15 \%$ had a cumulative dose of $>100 \mathrm{mSv}$. $^{9}$

Multiple techniques are available to reduce the radiation dose from CT scans, particularly to radiosensitive tissues such as the lens of the eye and the thyroid gland. These include iterative reconstruction and organ-based dose-reduction algorithms specifically targeting the lens and thyroid glands. There is evidence that 
these strategies can result in moderate dose reduction (up to $50 \%)^{10}$ without compromising image quality. ${ }^{11,12}$ MR imaging offers an imaging solution that uses nonionizing radiation, eliminating radiation dose concerns associated with medical imaging.

While most acute neurologic encounters in the ED can be assessed with CT, MR imaging offers a more detailed assessment, given its superior soft-tissue contrast, without using ionizing radiation. Apart from trauma and conductive hearing loss, the Appropriateness Criteria of the American College of Radiology list MR imaging as the preferred examination for most neurologic symptoms. These include ataxia, cranial neuropathies, dementia, altered mental status, focal neurologic deficits, headache (except thunderclap headaches), seizure, and vision loss. ${ }^{13}$

MR imaging and specifically diffusion-weighted imaging have been shown to be more sensitive in diagnosing acute stroke compared with CT. ${ }^{14}$ Chalela et al ${ }^{15}$ conducted a prospective, blinded comparison of MR imaging and CT for the assessment of acute stroke in the emergency department and concluded that MR imaging was superior to CT for the detection of acute ischemia and could detect acute and chronic hemorrhage, suggesting that MR imaging should be the preferred test for the accurate diagnosis of patients with suspected acute stroke. Other studies have confirmed that MR imaging is as accurate as CT for acute hemorrhage and more accurate for chronic hemorrhage. ${ }^{16,17}$ Indeed, evidence suggests that the increased sensitivity of MR imaging for intracranial blood, and particularly microbleeds, may help identify those patients at higher risk for secondary intracerebral hemorrhage when considering intravenous thrombolytic therapy. ${ }^{18,19}$

Growing evidence suggests that the combination of fluid-attenuated inversion recovery pulse sequences and diffusionweighted imaging is useful in determining which patients with strokes and unknown time of symptom onset or wake-up strokes are within the therapeutic window at presentation. ${ }^{20-23}$ Triaging patients with stroke to MR imaging instead of CT results in a better safety profile and a higher chance of favorable outcome when treating beyond 3 hours of symptom onset. ${ }^{24}$

When evaluating patients for possible endovascular therapy, contrast-enhanced MR angiography has been shown to be accurate in detecting stenosis of the carotid ${ }^{25}$ and vertebrobasilar circulations $^{26}$ and correlates well with digital subtraction angiography for carotid stenosis. ${ }^{27}$ Contrast-enhanced MRA has also been shown to be equivalent or superior to time-of-flight angiography with much shorter scan times ${ }^{28,29}$ and to improve detection of carotid terminus occlusion. ${ }^{30}$

Although MR imaging is not the primary technique for assessing trauma, mounting evidence demonstrates that MR imaging offers considerable value as an ancillary test in the acute phase. Fluid-attenuated inversion recovery sequences have been shown to be equivalent or better than CT for the detection of acute subarachnoid hemorrhage, ${ }^{31}$ with increased sensitivity for acute and subacute hemorrhage with the addition of $\mathrm{T} 2^{*}$ sequences. ${ }^{32,33}$ MR imaging has also been shown to be superior to CT in detecting diffuse axonal injury. ${ }^{34-36}$ In acute spinal trauma, Morais et $\mathrm{al}^{37}$ demonstrated that MR imaging is superior to CT for the detection of bone swelling, posterior ligamentous complex injury, disc herniation, cord compression, and cord swelling.

\section{Program Development}

For use of MR imaging in the ED to achieve maximal success, it was thought that the turnaround times for MR imaging needed to be as close to CT as possible. Multiple factors, from placement of the equipment through training of technologists, were optimized to maximize efficiency and patient throughput.

The location of the MR imaging scanner within the ED itself was an extremely important achievement in the development of a viable ED MR imaging program because it permitted simplification of workflow from the multitransfer inpatient workflow to a no-transfer workflow, which more closely resembles the ED CT process.

Before the installation of the MR imaging scanner within the $\mathrm{ED}$, patients requiring $\mathrm{MR}$ imaging would be entered into the hospital transport queue and would be taken to the inpatient scanners, accompanied by the responsible ED nurse. Once arriving at the inpatient scanner, care would be transferred to the MR imaging nurse for the duration of the scan, with all the potential delays that are inherent in any transfer-of-care process. Following the patient's scan, this process was repeated in reverse, essentially doubling the potential for delays.

When the MR imaging scanner was placed in the ED, the use of the hospital transport queue was eliminated, with patients being moved by the dedicated ED transport system for more efficient transport. Both transfers of care were eliminated because the ED nurses are able to maintain care of their patients throughout the MR imaging process. Additionally, because the scanner was placed in the ED, only patients who would require nursing support for ED CT would require nursing support for ED MR imaging. These alterations essentially created an MR imaging workflow that was identical to the existing CT workflow, markedly reducing the previous temporal disadvantage of MR imaging. A comparison of the current and prior workflows can be seen in the Figure.

The next portion of the care process that required alteration was the scan itself. Barrow Neurological Institute inpatient MR imaging protocols last from 30 minutes to $>1$ hour; this timeframe is not conducive to high patient throughput. New ED scan protocols were created to limit scan time while providing adequate information to make emergent treatment and admit/discharge decisions. These protocols were designed to be completed in 10 minutes. A full listing of the sequences is detailed in the Table.

These new workflows and scan sequences necessitated education of referring physicians, nursing services, and technologists because they were substantially different from the inpatient protocols they were replacing. To this end, multiple meetings were held with the emergency physicians and the inpatient services (predominantly neurosurgery and neurology) before launch. Discussions were also held with the neurosurgery residents, nursing services, and technologists. The emergency physicians as a group were generally willing to transition from CT to MR imaging if the turnaround times would indeed be similar. There was some initial concern from the ED nurses that they would be forced to be away from their other patients for extended lengths of time. The nursing service was skeptical about the claim that the turnaround times for patients would be similar to those of CT. The inpatient services also expressed concern with the new processes, partic-

AJNR Am J Neuroradiol 38:1480-85 Aug 2017 www.ajnr.org

1481 

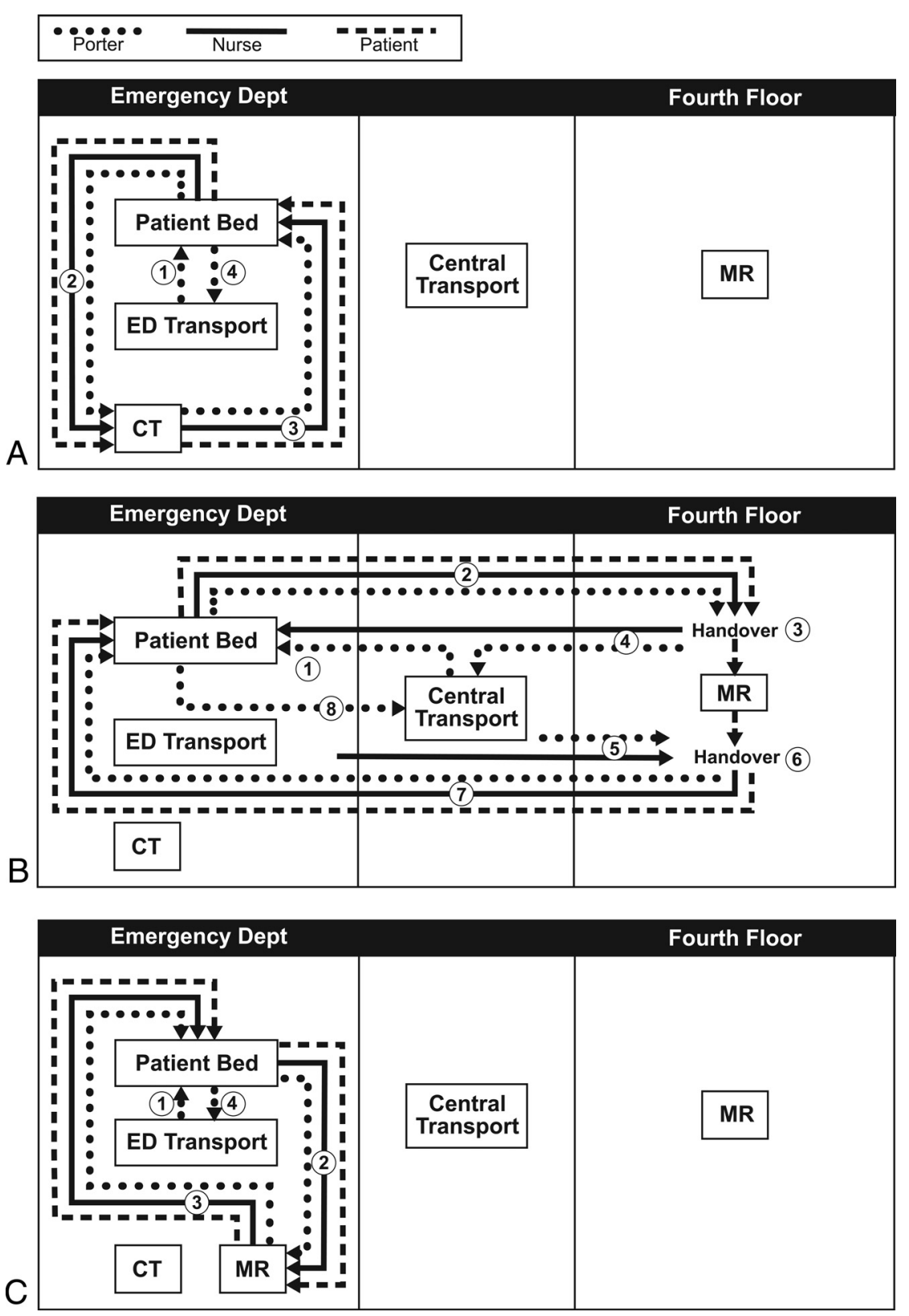

FIGURE. Workflow diagram. A, ED CT workflow: the ED porter collects the patient (1); porter, patient, and nurse go to CT (2); porter, patient, and nurse go to the patient's bed (3); porter goes to ED Transport (4). B, Prior MR workflow: central porter collects the patient (1); porter, patient, and nurse go to MR imaging (2); nursing handover with MR imaging nurses (3); nurse goes to ED, porter goes to Central Transport (4); ED nurse and central porter go to MR imaging (5); nursing handover with MR imaging nurses (6); porter, patient, and nurse go to patient's bed (7); porter goes to Central Transport (8). C, ED MR imaging workflow: ED porter collects the patient (1); porter, patient, and nurse go to ED MR imaging (2); porter, patient, and nurse go to patient's bed (3); porter goes to ED Transport (4).

ularly relating to the loss of information secondary to the limited protocols. It was emphasized that these scans were for emergent treatment and admit/discharge decisions and that any additional information required could be obtained with an inpatient scan should the patient in question require admission.

\section{Program Deployment}

The installation of the MR imaging scanner in the ED took approximately 2 years from the time it was initially approved. This process was substantially expedited because the ED had preexisting space available to house the scanner, avoiding extensive renovations/additions to the existing structure of the ED. Overall, there were no lengthy or unexpected delays, and the scanner went live in May 2015.

The new scan protocols were deployed in 2 phases. In phase 1, ordering guidelines were created with MR imaging replacing CT of the head as the primary imaging technique for all nontrauma, nonstroke indications in the ED. This phase was initiated in May 2015. Phase 2 was initiated in July 2016, when MR imaging of the head and contrast-enhanced MRA of the head and neck replaced CT of the head and CT angiography of the head and neck as the primary imaging technique for acute stroke. Scan data were collected from August through November in 2015 and 2016 and were compared with baseline data collected from August through November in 2014. Statistical significance was calculated by using the $\chi^{2}$ test. Results are reported as the percentage change between phases (number of CT scans/number of total scans, $P$ value).

Before the installation of the ED MR imaging scanner, 86\% (5538/6430) of the monthly scans performed in the emergency department were CT. Following initiation of phase 1 , there was a statistically significant drop in the percentage of CT studies to 62\% (4518/ 7288, $P<.001)$. The distribution of these CT scans changed slightly with fewer CT angiography studies (22\% to $15 \%)$ and an associated increase in the proportion of time-of-flight MRAs (5\% to $13 \%)$. There was also a large increase in the percentage of unenhanced MR imaging of the brain ( $17 \%$ to $29 \%$ ).

Following the initiation of phase 2, there was an additional statistically significant drop in the overall CT scan percentage to $48 \%(4161 / 8669, P<.001)$. The distribution of CT scans again changed slightly, with a continued decrease in the proportion of CTA studies (15\% to 9\%) and a marked increase in contrast-enhanced MRA, accounting for $11 \%$ of MR imaging studies. This change was associated with an increase in the proportion of enhanced MR imaging of the brain related to the new stroke protocol.

Throughput following phase 1 averaged between 25 and 26 scans per day during the 4 -month period. This number increased 
New ED scan protocols at Barrow Neurological Institute

\begin{tabular}{|c|c|}
\hline Scan & Sequences \\
\hline Unenhanced brain & Sagittal T1, axial DWI, axial FLAIR, axial T2 GRE \\
\hline Enhanced brain & $\begin{array}{l}\text { Sagittal Tl, axial DWI, axial FLAIR, 3D-T1 with and } \\
\text { without contrast }\end{array}$ \\
\hline Unenhanced $\mathrm{C} / \mathrm{T}$ spine & Sagittal T1, sagittal mDixon T2, axial T2 GRE \\
\hline Enhanced $\mathrm{C} / \mathrm{T}$ spine & $\begin{array}{l}\text { Sagittal Tl, sagittal STIR, sagittal and axial mDixon T1 } \\
\text { with contrast }\end{array}$ \\
\hline Unenhanced L spine & Sagittal T1, sagittal mDixon T2, axial T2 TSE, coronal T1 \\
\hline Enhanced L spine & $\begin{array}{l}\text { Sagittal T1, sagittal mDixon T2, axial T2 TSE, sagittal } \\
\text { and axial mDixon T1 with contrast }\end{array}$ \\
\hline Unenhanced MRA & Axial 2D TOF of the neck, 3D TOF of the head \\
\hline Enhanced MRA & 3D bolus dynamic contrast-enhanced \\
\hline
\end{tabular}

Note:-GRE indicates gradient-echo; mDixon, modified Dixon; C, cervical; T, thoracic; L, lumbar. the new sequences. A good example of these concerns is the decreased sensitivity of contrast-enhanced MRA for aneurysms. There were also concerns raised that the new, limited examinations would not meet the expectations of referring physicians and patients who expect a thorough and detailed examination from a specialized center like the Barrow Neurological Institute. In response to these concerns, statements detailing the purpose and limitations of the new ED protocols were included in reporting templates for these studies.

The effects on referring physicians were also varied. ED physicians have been satisfied with the turnaround and throughput and have expressed greater confidence with decisions to both admit and discharge patients. Concerns from inpatient services over missing diagnostic information have translated into persistent ordering of time-intensive scans despite the prelaunch education. These scans generally come in 2 variations: either large-area scans such as MR imaging of the brain and cervical, thoracic, and lumbar spine with and without contrast for investigation of multiple sclerosis, or small-FOV, thin-section imaging such as pituitary or orbit scans. Continued effort to emphasize the purpose of the ED scans is ongoing, including collaborative research projects to establish the value of certain scans in admit/discharge decisions. During our data collection, there were no instances of patients being recalled for additional images due to insufficient diagnostic information.

While the challenges with respect to the day-to-day ordering trends of the inpatient services were anticipated, the effects on the learners, particularly the neurosurgery residents, were not. Through hours of on-call work, the neurosurgery residents at Barrow Neurological Institute had become extremely proficient in extracting the information they needed to make decisions on call from inpatient MR imaging sequences with which they were familiar. The addition of ED MR imaging scan protocols optimized to reduce scan time replaced these familiar sequences with new sequences and severely undermined the residents' confidence in their on-call decision-making. The magnitude of this disruption was much greater than anticipated before launch and required additional education sessions with the neurosurgery residents to familiarize them with the new scan protocols and draw parallels between the sequences they knew and the corresponding new sequences. Provided with this foundation, the residents acclimated within 2-3 months to the new protocols and concerns regarding the ED scanner became infrequent.

\section{Future Directions}

Continued evaluation and improvement of the ED MR imaging workflows and protocols are ongoing to ensure that patients in the $\mathrm{ED}$ receive the maximal benefit from their imaging study. An example of this improvement is the development of a new $\mathrm{T} 2{ }^{\star}$ weighted sequence to replace the currently used gradient-echo sequence in the MR imaging brain protocol, which will provide improved spatial resolution with approximately 60-second shorter scan times. Additional fine-tuning of the protocols will 
continue as more experience is gained with a dedicated ED MR imaging scanner.

There are currently no plans to add additional MR imaging scanners to the emergency department. However, 2 new inpatient MR imaging scanners are scheduled to be installed adjacent to the ED, which will allow decompression of the ED scanner should ED scan volumes continue to rise and outstrip current capacity.

While we were able to significantly reduce the proportion of CT imaging studies ordered in the ED ( $86 \%$ to $48 \%$, a 56\% relative reduction), the overall volume of imaging rose continuously during the 2-year period, from 6430 per 4-month period in 2014, to 7288 in 2015, and 8669 in 2016 . Based on this rise, the absolute reduction in CT scans performed was 1379 studies per 4-month period, a $25 \%$ reduction. Concern has been raised that the installation of an MR imaging scanner in the ED has simply replaced inappropriately ordered CT scans with inappropriately ordered MRIs and that the underlying problem of rising use remains unaddressed. This argument merits attention, and other interventions such as persistent physician education, clinical decision support, updated guidelines, and pay-for-performance systems have been shown to be effective in decreasing use in academic centers. ${ }^{3,4}$ However, the issue of increased use is multifactorial, and a permanent solution would likely require systematic changes to payment structures and extensive alterations in the medicolegal landscape, both of which are extremely complex environments. Fast access to MR imaging from the ED has, at least in the interim, provided a safer and diagnostically superior replacement for CT.

\section{CONCLUSIONS}

The installation of a dedicated ED MR imaging scanner significantly reduced the number of CT scans performed in the ED by providing a diagnostically superior and safer imaging alternative with a drastically improved turnaround time. This was achieved by altering the MR imaging workflow and existing scan protocols with an emphasis on efficiency and patient throughput. This change has resulted in increased confidence in clinical decision-making for the ED physicians and, with the exception of the unanticipated severity of the culture shift on the MR imaging technologists and neurosurgery residents, has been relatively well-received. Whether this change has resulted in improved patient outcomes was not addressed and further investigation is required.

\section{ACKNOWLEDGMENTS}

We thank Kevin King and Jana Mahl for their tireless assistance with data collection for this project.

\section{REFERENCES}

1. Levin DC, Rao VM, Parker L, et al. Continued growth in emergency department imaging is bucking the overall trends. J Am Coll Radiol 2014;11:1044-47 CrossRef Medline

2. Rankey D, Leach JL, Leach SD. Emergency MRI utilization trends at a tertiary care academic medical center: baseline data. Acad Radiol 2008; 15:438-43 CrossRef Medline

3. Arasu VA, Abujudeh HH, Biddinger PD, et al. Diagnostic emergency imaging utilization at an academic trauma center from 1996 to 2012. J Am Coll Radiol 2015;12:467-74 CrossRef Medline

4. Raja AS, Ip IK, Sodickson AD, et al. Radiology utilization in the emergency department: trends of the past 2 decades. AJR Am J Roentgenol 2014;203:355-60 CrossRef Medline
5. Ahn S, Kim WY, Lim KS, et al. Advanced radiology utilization for a tertiary care emergency department from 2001 to 2010. PLoS One 2014;9:e112650 CrossRef Medline

6. Brenner DJ, Hall EJ. Computed tomography: an increasing source of radiation exposure. N Engl J Med 2007;357:2277-84 CrossRef Medline

7. Smith-Bindman R, Lipson J, Marcus R, et al. Radiation dose associated with common computed tomographic examinations and the associated life-time attributable risk of cancer. Arch Intern Med 2009;169:2078-86 CrossRef Medline

8. Berrington de González A, Mahesh M, Kim KP, et al. Projected cancer risks from computed tomographic scans performed in the United States in 2007. Arch Intern Med 2009;169:2071-77 CrossRef Medline

9. Sodickson A, Baeyens PF, Andriole KP, et al. Recurrent CT, cumulative radiation exposure and associated radiation-induced cancer risks from CT of adults. Radiology 2009;251:175-84 CrossRef Medline

10. Zhang WI, Li M, Zhang B, et al. CT angiography of the head-andneck vessels acquired with low tube voltage, low iodine, and iterative image reconstruction: clinical evaluation of radiation dose and image quality. PLoS One 2013;8:e81486 CrossRef Medline

11. Reimann AJ, Davison C, Bjarnason T, et al. Organ-based computed tomographic $(\mathrm{CT})$ radiation dose reduction to the lenses: impact on image quality for CT of the head. J Comput Assist Tomogr 2012;36: 334-38 CrossRef Medline

12. Ketelsen D, Buchgeister M, Fenchel M, et al. Automated computed tomography dose-saving algorithm to protect radiosensitive tissue: estimation of radiation exposure and image quality considerations. Invest Radiol 2012;47:148-52 CrossRef Medline

13. American College of Radiology: Appropriateness Criteria. https:// acsearch.acr.org/list. Accessed December 27, 2016

14. Fiebach JB, Schellinger PD, Jansen O, et al. CT and diffusionweighted MR imaging in randomized order: diffusion-weighted imaging results in higher accuracy and lower interrater variability in the diagnosis of hyperacute ischemic stroke. Stroke 2002;33: 2206-10 CrossRef Medline

15. Chalela JA, Kidwell CS, Nentwich LM, et al. Magnetic resonance imaging and computed tomography in emergency assessment of patients with suspected acute stroke: a prospective comparison. Lancet 2007;369:293-98 CrossRef Medline

16. Kidwell CS, Chalela JA, Saver JL, et al. Comparison of MRI and CT for detection of acute intracerebral hemorrhage. JAMA 2004;292: 1823-30 CrossRef Medline

17. Fiebach JB, Schellinger PD, Gass A, et al. Stroke magnetic resonance imaging is accurate in hyperacute intracerebral hemorrhage: a multicenter study on the validity of stroke imaging. Stroke 2004;35: 502-06 CrossRef Medline

18. Dannenberg S, Scheitz JF, Rozanski M, et al. Number of cerebral microbleeds and risk of intracerebral hemorrhage after intravenous thrombolysis. Stroke 2014;45:2900-05 CrossRef Medline

19. Kidwell CS, Saver JL, Villablanca JP, et al. Magnetic resonance imaging detection of microbleeds before thrombolysis: an emerging application. Stroke 2002;33:95-98 CrossRef Medline

20. Thomalla G, Cheng B, Ebinger M, et a; STIR and VISTA Imaging Investigators. DWI-FLAIR mismatch for the identification of patients with acute ischaemic stroke within $45 \mathrm{~h}$ of symptom onset (PRE-FLAIR): a multicenter observational study. Lancet Neurol 2011;10:978-86 CrossRef Medline

21. Thomalla G, Rossbach P, Rosenkranz M, et al. Negative fluid-attenuated inversion recovery imaging identifies acute ischemic stroke at 3 hours or less. Ann Neurol 2009;65:724-32 CrossRef Medline

22. Aoki J, Kimura K, Iguchi $Y$, et al. FLAIR can estimate the onset time in acute ischemic stroke patients. J Neurol Sci 2010;293:39-44 CrossRef Medline

23. Petkova M, Rodrigo S, Lamy C, et al. MR imaging helps predict time from symptom onset in patients with acute stroke: implications for 
patients with unknown onset time. Radiology 2010;257:782-92 CrossRef Medline

24. Schellinger PD, Thomalla G, Fiehler J, et al. MRI-based and CTbased thrombolytic therapy in acute stroke within and beyond established time windows: an analysis of 1210 patients. Stroke 2007; 38:2640-45 CrossRef Medline

25. Randoux B, Marro B, Koskas F, et al. Carotid artery stenosis: prospective comparison of $\mathrm{CT}$, three-dimensional gadolinium-enhanced MR, and conventional angiography. Radiology 2001;220: 179-85 CrossRef Medline

26. Yang CW, Carr JC, Futterer SF, et al. Contrast-enhanced MR angiography of the carotid and vertebrobasilar circulations. AJNR Am J Neuroradiol 2005;26:2095-101 Medline

27. U-King-Im JM, Trivedi RA, Cross JJ, et al. Measuring carotid stenosis on contrast-enhanced magnetic resonance angiography: diagnostic performance and reproducibility of 3 different methods. Stroke 2004;35:2083-88 CrossRef Medline

28. Nael K, Meshksar A, Ellingson B, et al. Combined low-dose contrastenhanced MR angiography and perfusion for acute ischemic stroke at 3T: a more efficient stroke protocol. AJNR Am J Neuroradiol 2014; 35:1078-84 CrossRef Medline

29. Alfke K, Jensen U, Pool C, et al. Contrast-enhanced magnetic resonance angiography in stroke diagnostics: additional information compared with time-of-flight magnetic resonance angiography? Clin Neuroradiol 2011;21:5-10 CrossRef Medline

30. Saager C, Fitting T, Goebell E, et al. Contrast-enhanced MR angiography improves detection of carotid T-occlusion by acute stroke MRI. Clin Neuroradiol 2008;18:163-67 CrossRef
31. da Rocha AJ, da Silva CJ, Gama HP, et al. Comparison of magnetic resonance imaging sequences with computed tomography to detect low-grade subarachnoid hemorrhage: role of fluid-attenuated inversion recovery sequence. J Comput Assist Tomogr 2006;30:295-303 CrossRef Medline

32. Yuan MK, Lai PH, Chen JY, et al. Detection of subarachnoid hemorrhage at acute and subacute/chronic stages: comparison of four magnetic resonance imaging pulse sequences and computed tomography. J Chin Med Assoc 2005;68:131-37 CrossRef Medline

33. Verma RK, Kottke R, Andereggen L, et al. Detecting subarachnoid hemorrhage: comparison of combined FLAIR/SWI versus CT. Eur J Radiol 2013;82:1539-45 CrossRef Medline

34. Paterakis K, Karantanas AH, Komnos A, et al. Outcome of patients with diffuse axonal injury: the significance and prognostic value of MRI in the acute phase. J Trauma 2000;49:1071-75 CrossRef Medline

35. Lee H, Wintermark M, Gean $\mathrm{AD}$, et al. Focal lesions in acute mild traumatic brain injury and neurocognitive outcome CT vs 3T MRI. J Neurotrauma 2008;25:1049-56 CrossRef Medline

36. Mittl RG, Grossman RI, Hiehle JF, et al. Prevalence of MR evidence of diffuse axonal injury in patients with mild head injury and normal head CT findings. AJNR Am J Neuroradiol 1994;15:1583-89 Medline

37. Morais DF, de Melo Neto JS, Meguins LC, et al. Clinical applicability of magnetic resonance imaging in acute spinal cord trauma. Eur Spine J 2014;23:1457-63 CrossRef Medline 